Review

\title{
Unmanned Aerial Systems (UAS) Research Opportunities
}

\section{Javaan Chahl ${ }^{1,2}$}

${ }^{1}$ School of Engineering, University of South Australia, Adelaide 5001, Australia;

E-Mail: javaan.chahl@unisa.edu.au; Tel.: +61-(0)-883-026-803

2 Joint and Operations Analysis Division, Defence Science and Technology Organisation, Canberra 2601, Australia

Academic Editor: Rafic Ajaj

Received: 30 January 2015 / Accepted: 20 April 2015 / Published: 27 April 2015

\begin{abstract}
The aerospace community is planning for growth in Unmanned Aerial Systems (UAS) funding and research opportunities. The premise that UAS will revolutionize aerospace appears to be unfolding based on current trends. There is also an anticipation of an increasing number of new platforms and research investment, which is likely but must be analysed carefully to determine where the opportunities lie. This paper draws on the state of technology, history and systems engineering. We explore what aspects of UAS will be the result of aerospace science advances and what aspects will be incremental engineering and systems integration. It becomes apparent that, for academia, the largest opportunities may exist in small and micro UAS domain due to the novelty of aerospace engineering on a small scale.
\end{abstract}

Keywords: unmanned aerial system (UAS); unmanned aerial vehicle (UAV); research; defense

\section{Introduction}

Expectations of growth in the Unmanned Aerial Systems (UAS) industry are quite high, Teal Group predicted that "Unmanned Aerial Vehicles (UAVs) continue as the most dynamic growth sector of the world aerospace industry this decade" [1]. This is despite the United States Department of Defence (US DoD), the largest single customer for UAS [2], projecting reduced funding for Research, Development, Testing and Evaluation [2] of UAS. In an environment containing both growth in inventory and reduction in research funds it is important to understand which technologies are absolutely required for progress and which technologies are likely to be advanced by other disciplines outside aerospace. 
Like any other complex system, UAS are composed of a series of major subsystems. Many of these subsystems are common to other aerospace, communications and intelligence, surveillance and reconnaissance (ISR) systems. Whilst enhancements in these systems will improve UAS performance, they might not enable new capabilities, except where thresholds of specific subsystem parameters must be achieved to undertake a new mission or role. In this paper we will attempt to demonstrate where the core of UAS engineering research lies. We will also outline those areas that require a threshold of functionality or size to affect change in UAS roles.

The United States Department of Defense use the following definitions [3]:

"Unmanned aircraft-An aircraft that does not carry a human operator and is capable of flight with or without human remote control. Also called UA."

"Unmanned aircraft system-That system whose components include the necessary equipment, network, and personnel to control an unmanned aircraft. Also called UAS."

Aboard the UAS are a control system, payloads, communications and a number of other subsystems that conceptually map to standard air vehicle subsystems. It is arguable that, as with a manned system, the ground station or command control element is not really integral to the system or necessary when UAS are more autonomous. The software of the UAS guidance system consists of control systems for stabilisation and navigation, which we will describe as "control", and a degree of decision making and sensor processing that we shall describe as "Autonomy". The payloads are sometimes UAS specific and are generally used operationally in such a way that payload implementations dominate the role of the entire system.

This paper is focused on UAS platform technology. There are many technologies loosely related to high levels of autonomy that could be considered, however, for aerospace vehicle research, the design, performance and control of the air frame to meet requirements is the the emphasis.

UAS only became truly effective with the advent of GPS, previous limited successes navigated using high quality inertial measurement units, terrain registration or line of sight video telemetry. The Teledyne-Ryan Firefly and descendant target drones modified by the US Air Force for reconnaissance during the Vietnam War era [4] were conceptually a successor to the high performance Lockheed-Martin D-21 [5]. Navigating on inertial and air-data systems these drones were capable of occasionally capturing imagery from inside heavily defended airspace. GPS has subsequently provided the means for aircraft to be unattended for long periods of time, even the entire mission. Remotely Piloted Vehicles (RPVs) have generally provided a capability only in limited missions, such as being a target. Oddly a very early rotary wing UAV, the Gyrodyne QH-50 DASH achieved a degree of success in the Vietnam war era [6] probably due to its high degree of stabilisation and ability to fly slowly and hover.

The aerial domain is generally uncluttered compared to ground environments. Many of the signals used to make decisions are quite simple and artificially derived, for example weather radar, GPS and transponders. For some decades autopilots have been able to fly aircraft that no human could fly [7-9], so the autonomy problems associated with mobility have been overcome for most situations. 
The types of objects present in the high altitude aerial domain are limited to other aircraft. Avoiding, docking with and engaging other aircraft are much more constrained problems than the difficult situations a ground robot might encounter in any patch of woodland.

Whenever the environment becomes complex, UAS autonomy requirements become demanding. Launch and recovery are perennial problems for UAS, not only due to normal air frame limitations, but because of the sensing and reasoning required for operation on or near the ground. For the same reason, vision based nap of the earth flight is a challenge to perform autonomously because of the processing and decision making required. Vision for terrain following and collision avoidance is still a research problem $[10,11]$.

Small and micro UAS will face larger autonomy challenges than large UAS due to the expectation that they will operate in and around obstacles [12]. Flight through clutter by a space craft such as a UAV is easier from an autonomy and platform perspective than crawling through it. It would be expected that the craft would not touch the environment, or need to climb along it, thus path planning is relatively simpler, but ultimately more limiting than a robot crawling through very dense clutter.

It is probable that in the future UAS will be required to engage in the full spectrum of air operations. In these scenarios the complexity of the task and the environment will require higher levels of automation than are found in current UAS [13].

\section{Classes of UAS from An Aerospace Perspective}

These definitions will be used for the purposes of this analysis. We will focus on the primary challenges to the advancement of each class. Weight is a significant discriminator between UAS types as is role. UAS class definition is a multidimensional taxonomic problem that has been collapsed into one dimension by bureaucracies with resulting loss of meaning. It is indicative that the very name UAS indicates what the aircraft is not (not manned), rather than what the technology aspires to be (autonomous). For the purpose of this analysis we will use the US DoD "Group" nomenclature [2].

\subsection{Micro UAS}

We will assume that micro UAS are represented at the large end by the Aerovironment Wasp AE [14] and at the lowest end, as of this writing, by the Proxdynamics Black Hornet [15]. A legal definition exists in Australian Civil Aviation Regulations [16], which imposes a $100 \mathrm{~g}$ weight limit, below which systems are defined as a "micro UAV" and not regulated. There are numerous programs to reduce the size of UAS well below the Black Hornet's $18 \mathrm{~g}$. Currently there is nothing acknowledged that is smaller and claimed to have achieved operational utility.

In the currently dominant military and law enforcement contexts, very small UAS are expected to be useful for a range of ISR tasks, including: personal ISR by individual infantry, counter terrorism and law enforcement personnel; base and airfield surveillance; detection of hazardous materials.

Other roles are speculative and there is increasing interest from a range of potential users including the media, but at this scale payload capability is necessarily limited. Other roles will develop as the systems become better understood and emerge from the current state. Generally, the small size of the aircraft satisfies requirements for convenience and limited carriage/stowage burden and offers the promise of 
mission agility (quick to deploy) and physical agility (the ability to manoeuvre in small spaces such as narrow streets and even inside rooms). Critically, these systems offer the promise of safe operation, for example, operation in a metropolitan area over civilians when threat levels are too low to justify the hazard presented by a large high capability UAS.

These systems are limited as a direct result of their size. It is clear that multidisciplinary technical hurdles are non-trivial and functional limitations are unavoidable.

The low Reynolds number domain requires specialized airfoils and wing shapes [17]. This regime may favour flapping wing flight with interactions between aerodynamic surfaces and vortices [18], once efficiency of the aero-electro-mechanical system is understood and improved [19]. Currently there is little concrete progress towards a practical flapping wing UAS.

Performance improvements will be driven by traditional aeronautical research. The empirical approach of hobbyists and the market driven engineering of small companies providing hobby components may also play a role. Performance improvements are multiplicative for flight systems, such as wing aerodynamics and propeller efficiency, so a series of modest optimizations of different systems might increase performance substantially.

Steering, pointing and zooming of electro-optical (EO) payloads is limited by the size of robust, accurate mechanics. This limit is constrained by the precision achievable in mass produced items. Other issues include optical limits set by lens size and focal plane size. There is one main market driving small EO payloads: mobile telephones. Features such as face tracking and internal zoom mechanisms can be directly applied to UAS applications. High resolution thermal cameras, laser radar and other sensors found in larger UAS are currently a challenge for micro UAS. This could change if these sensors become part of mass market products such as mobile telephones.

The physical size of guidance hardware (the autopilot) is a problem in this class. Advances in micro-electro-mechanical systems (MEMS) and their integration into multisensor integrated circuits is resulting in dramatically decreased size.

A notable deficiency in all UAS to date is the Human Machine Interface (HMI), this is particularly the case in the micro UAS domain. A significant conceptual advance is required, possibly including display technologies which will improve continuously thanks to mobile devices. It seems reasonable that the human interface should be proportionate to the size of the air vehicle. Ultimately HMI should use natural human communication and largely eliminate the ground control element.

\subsection{Group 1: Organic UAS}

The next class of UAS includes Lockheed Martin's Desert Hawk [20], Aerovironment's Raven [21], and Elbit's Skylark [22] among many less commercially successful types. UAS in this class compete with each other on payload capability, endurance and recovery options. Miniaturization and demonstrated value of the class should ensure that capability of platforms and payloads will increase to the limits of feasibility.

Generally this class will be launched by hand, bungee or highly portable launch apparatus. The ground element will be a small computer and some portable telemetry equipment. Crew consist of an individual or a small team. Specialization of the team members for UAS operations is relatively 
low. Design improvements and set-up/tear-down effort will be differentiators between systems in this class as much as payload capability. Research in this Group will be dominated by attempts to achieve the endurance and payload capabilities of larger UAS.

This class has some overlap with micro UAS. Scope for platform improvement is limited, since many of the subsystems are close to theoretical limits of efficiency due to the manufacturability of the scale of subsystems and demand from the hobby market and Defense for portable devices in this size range. Energy density of batteries will remain an issue.

Challenges will continue to include improved thermal focal plane arrays, miniature gimbaled cameras and inertial measurement units capable of aiding geolocation and navigation. Continued investment means that these payloads are becoming common at this scale. Automatic ISR functionality will be of interest because of the small crew and non-specialization of the users. Mechanical design and reliability will remain operational concerns because the systems are too large to rely solely on small size for robustness. The result is hard landings and attrition which can be addressed by design and flight control [23].

The greatest movement in this field is the use of commercial and partly commercial platforms with open source autopilots for aerial photography and surveying. The use of these systems by the public has become so widespread that difficulties in maintaining exclusive access to airspace for authorities during emergencies is becoming difficult [24]. The pace of development within the open source autopilot movement is so fast that many functions necessary to market a personal UAS to the non-enthusiast consumer will be completed within months. The Ardupilot Mega [25] and Pixhawk [26] hardware stand out in this regard as capable automation systems for Group 1 civilian UAS. At the time of this writing a simple Tier 1 UAS capable of transmitting imagery can be assembled for $\$ 500$ US. There has also been extensive discussion of using UAS for delivery of small freight [27].

\subsection{Group 2 to Small Group 3: Tactical UAS}

This class of UAS is used for tactical ISR, with increasing options to do targeting. Launch is usually from a rail with landing not directly onto the ground due to the need for delicate payloads and to maintain tactical mobility which precludes dependency on an undercarriage or runway.

Aircraft in this group include ScanEagle [28], Aerosonde [29] and Bat [30], with smaller Group 3 systems like Shadow 200 [31] at the upper end. UAS aeronautical issues in this domain are primarily engineering, with very little opportunity for science and minor opportunities for technology improvements. Despite their relatively small size, development has still required significant funds (typically many millions) and has largely been the domain of Defense. Early rapid acquisitions have given way to long term solutions procured using traditional Defense acquisition methods.

Challenges in this domain include: Recovery as a combination of control, sensing and navigation; sense and avoid as a combination of the science of sensing and process to achieve certification; improvements of currently marginal thermal imaging systems; robust and compact ISR payloads. 


\subsection{Large Group 3 to Group 5: Manned Aircraft-Sized UAS}

This class of UAS covers systems like the Hermes 450 [32] at the low end to MQ-9 Reaper [33] and RQ-4 Global Hawk [34]. There is significant commonality of all systems on these aircraft with manned systems. Advances in this domain will tend to involve autonomous operation of formerly manned activity. For example sense and avoid of air traffic, interactions with air traffic control, navigation and piloting. Size weight and power are unlikely to be larger issues than in comparable manned aircraft. Airworthiness and seamless integration with military and civilian airspace will be primary concerns.

Currently these systems use a large number of ground based personnel to manage the vehicle and analyze the ISR data [35]. Software developed to automatically seek salience in ISR data will aid in reducing the personnel requirements of larger UAS.

This group would also include converted air transports and other "optionally manned" concepts that may soon fulfill some commercial aviation roles [36].

Group 3 to Group 5 systems are larger and more expensive to develop and acquire than Group 2 systems, Defense are the dominant customer. They are moving from rapidly fielded and rapidly developing systems to more permanent and traditional acquisitions.

\subsection{Extreme Altitude (XA)}

It remains to be seen if this class eventuates as fielded systems. Their primary limitation is the simultaneous requirement to be extremely light for electric flight at high altitudes, while also performing a useful purpose.

There are a number of apparent challenges in this class, primarily related to energy constrained design for near-space conditions: Energy storage and management of payload, propulsion and charging systems; mechanical systems that will operate continuously in that environment possibly for years; light weight materials for airframe construction; integration of solar cells; design of more efficient low Reynolds number wings and propellers.

Even though the systems offer relatively low platform cost and potentially small footprint (and therefore low total cost), these systems still represent significant challenges in development and testing that do not necessarily yield a significant payoff. The prospects of these systems providing responsive communications infrastructure is starting to emerge, with Google among others apparently considering this application [37].

\subsection{Unmanned Combat Aerial Vehicles (UCAV)}

These systems are typified by a high requirement for autonomy, with a potentially contradictory requirement for weapons release to be under positive control by a human operator or supervisor, trust in the autonomy of the aircraft will be paramount. It includes the general topic of UAS in defended airspace. Primary research areas include: Dynamic path planning through threat fields; a general reduction in external dependencies such as data links and GPS; flight control for agility, low observability and efficiency; air to air combat; non-emitting nap of the earth flight; carrier landing; flush sensors and antennae and airframe signature reduction. 


\section{Fields of Innovation in UAS}

The table of contents of major aerospace conferences shows many UAS related papers. Of these papers many are platform oriented, based on the premise that UAS provide a less constrained solution space than manned aircraft. Yet, UAS platforms are consolidating in Groups 1 to 4 with very few basic planforms and minimal exotic aeronautics. Groups 2 to 4 UAS are simple aerodynamically compared to manned aircraft, as they are not fast, agile or large, or aggressively optimised for a well understood role (such as an airliner). There is no apparent drive to change this. The exceptions to this are Boeing/Insitu's ScanEagle [28] and Integrator/RQ-21A Blackjack [38], which won STUAS/Tier II competition [39] and Northrop Grumman Killer Bee/Bat [30]. ScanEagle, Bat and Integrator are unusual air frames, with unusual launch and recovery mechanisms. Only in the UCAV, XA and Group 1 and below are significant platform innovations visible or perhaps tolerated by stakeholders.

Many fielded UAS have clear road maps to 2020 and beyond [2], and new platforms are less favoured than upgrades to existing ones. Breakthroughs in research on air frame or propulsion design in Groups 2 to 4 UAS do not appear to be immanent.

With the exception of quadrotor aircraft, Group 1 show minimal platform innovation, indeed many Group 1 UAS trace their conceptual design to the late 1980s rudder steered glider, the Aerovironment Pointer [40].

It is illustrative to list a mixture of manned aircraft and unmanned aircraft, the innovation found in their design or system and the era in which the work was done, shown in Table 1.

Table 1. List of aircraft, the innovation with which they were equipped, and the approximate era.

\begin{tabular}{lll}
\hline Aircraft & Innovation & Era \\
\hline U-2 & Altitude, High Aspect Ratio Jet & 1955 \\
SR-71/D-21 & Manned/Unmanned Team, Speed, Gigh End UAS & 1960 \\
Harrier & VTOL & 1965 \\
F-111 & Variable Geometry, Escape Capsule, Turbofan, Terrain Following & 1965 \\
Firebee et al. & UAS, $c f$. D-21 & 1965 \\
F-16 & Fly by wire, Relaxed Stability & 1970 \\
Pioneer et al.. & First Tactical UAS & 1980 \\
F-117 & Stealth, Instability & 1980 \\
Pointer & Hand launch UAS, Deep Stall Recovery & 1986 \\
F-22 & Thrust vectoring, Aerodynamic Stealth, High Supercruise & 1990 \\
Global Hawk & $c f$. U-2, D-21 & 1995 \\
Scan Eagle & Sky Hook & 2002 \\
Quadrotor & Mechanically Simple VTOL & $c a .2000$ \\
\hline
\end{tabular}

Relative lack of innovation opportunity in UAS in Groups 1 to 4 is quite striking. UAS innovation is tending to occur in their operational application rather than their design. Operations then drive subsystem technologies to provide the required capability. To clarify the logic of what is transpiring: the golden age of innovation in air vehicles occurred in the 1940s to the 1960s; UAS are air vehicles, so it is unlikely that dramatic revolutions in air vehicle design will occur at intermediate scales. 
Operational success may have even stifled various elements of innovation as major UAS such as MQ-9 are still being procured and upgraded [41].

There is no lack of innovative thought in air vehicle science. It appears that the cost, risk or specialization associated with innovation is not justified in the current Defense UAS market. To date the strength of fielded UAS designs above Group 1 has been in their ability to be adapted to new roles, indicating a beneficial lack of specialization and thus lack of support for specific optimization.

\section{Specific to UAS Technology}

It is clear that an understanding of which research problems are specific to a class of UAS is required to determine how research resources should be invested and on which Groups research goals should be pursued in order to yield advances in the field.

It is also apparent that a class of UAS may improve because it relies on technology that is being improved by other fields but not driven by the UAS industry or academia. We will attempt to determine which technology areas are likely to need research and development targeted only at UAS.

Table 2 shows that the specific UAS problems are primarily in the domains of the micro UAS/Group 1, XA and UCAV.

Table 2. indicates that Research will significantly enhance a technology in a class of Unmanned Aerial Systems (UAS), indicates that some specific research is required, $\bigcirc$ indicates that Research will have little real impact.

\begin{tabular}{|c|c|c|c|c|c|c|}
\hline Subsystem & Micro UAS & Tier 1 & Groups 2-3 & Groups 3-5 & $\mathbf{X A}$ & UCAV \\
\hline GCS & D & D & 0 & $\bigcirc$ & 0 & $\bigcirc$ \\
\hline Data Links & D & 0 & 0 & 0 & 0 & 0 \\
\hline Air Vehicle & 0 & 0 & 0 & 0 & 0 & 0 \\
\hline Propulsion & 0 & 0 & 0 & 0 & D & 0 \\
\hline Payload & D & O & 0 & O & D & 0 \\
\hline Control & 0 & 0 & O & (1) & 0 & $\bullet$ \\
\hline Autonomy & 0 & 0 & D & D & 0 & 0 \\
\hline
\end{tabular}

The group with the largest number of unique research issues is micro UAS. Many of the systems must be smaller than available COTS systems, and some components might require advances in basic technologies such as materials and actuators. Burgeoning issues like flapping wing flight will need to be addressed as UAS approach the size and mass of insects, in which even the conceptual framework for design is the topic of research and debate. Many of these issues have no correlate to non UAS systems, and are not relevant to any other group of UAS. The operational demands for autonomy of small UAS appears to be higher than for autonomy in larger UAS. Collision avoidance, flight inside buildings and swarming are enduring concepts amongst funding agencies for over 15 years [12,42].

Groups 1 to 4 have few unique pressing aeronautical concerns that would be aided by research. Most of the subsystems that are critical to performance are driven as much by the manned aircraft industry, hobby industry or mass-market appliances. Much of the work will be normal product engineering. 
Group 1 has some overlap with micro UAS in needing acceptable payloads that challenge the size of consumer equipment, while not benefiting from manned aircraft ISR technology. Most payload issues are not within the narrow definition of aeronautics. Group 1 shares with micro UAS some of the problems of environmental hazards near the ground.

XA systems require substantial research to become practical. Improvement in bearings, actuators, structures and autonomous health monitoring for reliability and efficiency may need to be achieved. This class faces aerodynamic and structural challenges that generally also become control system problems due to the flexibility of the craft, such as the products of the NASA ERAST program [43]. The uncluttered, high altitude environment ensures that the need for advanced autonomy is quite low.

UCAV face a number of problems related to the antagonistic environment in which they operate that will inhibit data links and ISR functions and probably place additional reliance on autonomy. Robust control of unusually shaped aircraft remains an active area of scientific research. Although not generally publicized it seems safe to assume that advances in manned fighters and bombers are significant contributors to advances in UCAVs. This research is removed from the mainstream of academic and industry research. This assessment of current UCAV research needs is speculative at best.

A further consideration of micro UAS is necessary due to their apparently strong benefit from research. The primary driver of technology must be considered. In this consideration we are classifying the following potential actors in driving the state of the art forward. Commercial technology is what could be described as "General" technology for example that associated with telephones or composite materials. We designate the driver "Hobby" for developments created for hobby applications. "UAS" indicates research in institutions and industry is the natural driver.

Table 3. Strong drivers of technology advances are indicated with $\bullet$, weak drivers or highly applied research needs are indicated with $\mathbf{O}$, and drivers that are not likely to be effective with $\bigcirc$.

\begin{tabular}{|c|c|c|c|c|c|}
\hline Subsystem & Improvement & Discipline & General & Hobby & UAS \\
\hline Battery & Energy Density & Chemistry & 0 & $\bigcirc$ & $\bigcirc$ \\
\hline Propeller & Efficiency & Aerodynamics & 0 & $\mathbf{D}$ & 0 \\
\hline Wing & Efficiency & Aerodynamics & $\bigcirc$ & D & - \\
\hline \multirow[t]{2}{*}{ Motors } & Efficiency & Electromechanics & 0 & $\bigcirc$ & D \\
\hline & Reliability & Electromechanics & $\bigcirc$ & $\bigcirc$ & - \\
\hline \multirow[t]{2}{*}{ Actuators } & Robust Precision & Electromechanics & 0 & D & D \\
\hline & Miniaturization & Electromechanics & 0 & $\bullet$ & D \\
\hline Flapping & Miniaturization & Electromechanics & 0 & $\bigcirc$ & $\bullet$ \\
\hline \multirow[t]{3}{*}{ HMI } & Effectiveness & Software & O & (1) & D \\
\hline & Size & Hardware & - & (1) & $\bigcirc$ \\
\hline & Ergonomics & Human Factors & 0 & $\bigcirc$ & $\bigcirc$ \\
\hline Autonomy & Effectiveness & Software & O & O & - \\
\hline
\end{tabular}


This analysis is based on the historical contributions to these fields and the current level of investment. By far the most aerodynamics research on small wings and propellers happens in institutions funded by Government with UAS in mind. Batteries on the other hand are a technology of interest to the huge industry of mobile telephones and power tools. The research required to effectively use new sources of power in UAS is rather specific, but limited compared to actually inventing a power source. Most small UAS today have variations of commercial actuators adapted from the commercial hobby world and often electrically compatible with them. The micro UAS discipline moves further away from the hobby industry and towards UAV research programs when flapping wings [44,45] and extreme miniaturization [46] are involved. Autonomy is typically specific to the domain of UAS and mainly in institutions, although developments like the Robot Operating System [47] are causing a convergence of autonomy effort on problems rather than platforms and starting to involve effective hobbyists.

Historically human machine interfaces for UAS have been poorly done on both large and small UAS, with expensive lessons learned [48]. There are substantial research opportunities in this area.

\section{Discussion}

Mature, functional and affordable UAS are a comparatively new aerospace technology. For a time it was considered likely that an unbounded future of air vehicle design and innovation would emerge. The various government procurement processes have had the effect of rationalizing the field. The purchase price and operating cost of fielded systems to date has not encouraged an explosion of diversity. In general the cost model associated with aerial capabilities did not change with the advent of capable UAS [49].

Although government selected air frames may be difficult to supplant, opportunities do exist to improve navigation systems, aerodynamic models, control systems and avionics. The less stringent certification processes and absence of a human on-board do allow the scientific community to modify systems that are already in service [50], but this too is changing.

Smaller UAS research is the area where industry might be reluctant to invest substantial resources due to limited opportunities for profit. Micro UAS are also the area where the science community can safely develop and operate their own research capabilities. Large laboratories have formed around micro UAS and Group 1 UAS [51]. Diversity of procured and fielded systems is most likely to occur in this scale due to low past investment, low cost and low risk. Unlike the larger UAS, the micro UAS domain will continue to be strongly influenced by scientific research.

The question of scalability of UAS for missions becomes significant when micro UAS are considered. It appears that physical constraints create limits that can not be overcome. For example for high altitude ISR missions, satellite communications requires large steerable antennas; large apertures are needed for sensors; active sensors demand significant electrical power. In many large UAS, electrical power for payload is more at issue than mechanical power for manoeuvre. A deeper consideration would ask whether these missions are simply the result of the current paradigm. All of the physical constraints listed above can be overcome by distribution of sensors, emitters and receivers under a different operational concept. Outside the domain of information and data there are challenges that simply reflect the physics underpinning aerospace: a conventionally powered micro UAS will not have long range at high speed. 


\section{Conclusions}

We did not analyze what topics should be done in universities, by industry, or by Government laboratories. We did consider whether a particular area of research was UAS research, or really research into more general technology. We quickly identified that micro UAS as a technology can benefit most from scientific research, and speculate that university research can play a driving role due to low cost and low risk. Research on UCAV and XA craft might tend to be done between Government and Industry due to cost and complexity.

The different classes of UAS each have opportunities and risks for research investment. It is apparent that the greatest system level need for research exists at the extremes (micro, XA, UCAV) with the middle Groups having the least opportunity for major innovation that is specific to UAVs. It is probable that systems that do not fit the preconceived roles of classes of UAS will result in the largest return on investment. Understanding the customers and predicting what customers are likely to want is fundamental to obtaining ongoing support for well funded Aerospace research.

\section{Conflicts of Interest}

The author declares no conflict of interest.

\section{References}

1. Teal Group Predicts Worldwide UAV Market will Cost Total $\$ 89$ Billion in Its 2013 UAV Market Profile and Forecast. Available online: http://tealgroup.com/index.php/about-teal-groupcorporation/press-releases/94-2013-uav-press-release (accessed on 25 January 2015).

2. Winnefeld, J.A., Jr.; Kendall, F. DoD Unmanned Systems Integrated Roadmap FY2013-2038; Technical Report 14-S-0553; United States Department of Defence: Washington, DC, USA, 2013.

3. United States Department of Defence. Joint Publication 1-02: Department of Defense Dictionary of Military and Associated Terms; Technical Report JP1-02; United States Department of Defence: Washington, DC, USA, 2010.

4. Wagner, W. Fireflies and Other UAVs (Unmanned Aerial Vehicles); Aerofax Inc: Arlington, TX, USA, 1992.

5. Peebles, C. Dark Eagles; Presidio Press: Novato, CA, USA, 1999.

6. Maksel, R. D.A.S.H. goes to war. Air Space Mag, March 2012, p. dash-goes-to-war-23369442.

7. Edwards, C.; Lombaerts, T.; Smaili, H. Fault Tolerant Flight Control: A Benchmark Challenge; Springer: Berlin, Germany, 2010.

8. Sweetman, B. F-22 Raptor; Zenith Imprint: Minneapolis, MN, USA, 1998.

9. Clarke, R.; Burken, J.; Bosworth, J.; Bauer, J. X-29 flight control system: Lessons learned. Int. J. Control 1994, 59, 199-219.

10. Ruffier, F.; Franceschini, N. Optic flow regulation in unsteady environments: A tethered MAV achieves terrain following and targeted landing over a moving platform. J. Intell. Rob. Syst. 2014, doi:10.1007/s10846-014-0062-5.

11. Garratt, M.; Chahl, J. Visual control of an autonomous helicopter. In Proceedings of the 41st Aerospace Sciences Meeting and Exhibit, Reno, NV, USA, 6-9 January 2003. 
12. Fast Lightweight Autonomy (FLA). Available online: http://www.darpa.mil/Our_Work/DSO/ Programs/ Fast_Lightweight_Autonomy_\%28FLA\%29.aspx (accessed on 29 January 2015).

13. Pardesi, M.S. Unmanned aerial vehicles/unmanned combat aerial vehicles: Likely missions and challenges for the policy-relevant future. Air Space Power J. 2005, 45-54.

14. Aerovironment Inc. Wasp AE. Available online: http://www.avinc.com/uas/small-uas/waspAE/ (accessed on 20 January 2015).

15. Teeny Tiny Microcopter Deploys to Afghanistan with British Troops. Available online: http://spectrum.ieee.org/automaton/robotics/military-robots/microcopter-deploys-with-britishtroops (accessed on 15 April 2015).

16. Civil Aviation Safety Authority. Available online: http://www.casa.gov.au/scripts/nc.dll? WCMS:PWA::pc=PARTS101 (accessed on 15 April 2015).

17. Selig, M.; Donovan, J.; Fraser, D. Airfoils at Low Speeds; H. A. Stokely: Virginia Beach, VA, USA, 1989.

18. Ellington, C. The novel aerodynamics of insect flight: Applications to micro-air vehicles. J. Exp. Biol. 1999, 202, 3439-3448.

19. Kok, J.; Chahl, J. Systems-level analysis of resonant mechanisms for flapping-wing flyers. J. Aircraft 2014, 51, 1833-1841.

20. Lockheed Martin. Available online: http://www.lockheedmartin.com.au/us/products/deserthawk.html (accessed on 20 January 2015).

21. UAS: RQ-11B Raven. Available online: http://www.avinc.com/uas/small_uas/raven/ (accessed on 20 January 2015).

22. Elbit Systems. Skylark I LE-Mini UAS. Available online: https://www.elbitsystems.com/elbitmain/ area-in2.asp? parent=3\&num=279\&num2=279 (accessed on 20 January 2015).

23. Crowther, W.; Prassas, K. Post-stall landing for field retrieval of unmanned air vehicles. In Proceedings of the 14th Bristol International Unmanned Air Vehicle Systems Conference, Bristol, UK, 12-14 April 1999.

24. CFS to Ground Aerial Firefighting Fleet if a Drone Is Spotted Near a Bushfire. Available online: http://www.abc.net.au/news/2014-11-21/cfs-to-ground-aerial-firefighting-fleet-if-a-drone-isspotted/5909408 (accessed on 20 January 2015).

25. 3D Robotics. The APM Universal Autopilot. Available online: http://3drobotics.com/apm/ (accessed on 20 January 2015).

26. Meier, L.; Tanskanen, P.; Fraundorfer, F.; Pollefeys, M. PIXHAWK: A system for autonomous flight using onboard computer vision. In Proceedings of the IEEE International Conference on Robotics and Automation (ICRA), Shanghai, China, 9-13 May 2011; pp. 2992-2997.

27. Six things you should know about Amazon's drones. Available online: http://www.forbes.com/ sites/gregorymcneal/2014/07/11/six-things-you-need-to-know-about-amazons-drones/ (accessed on 20 January 2015).

28. Boeing. ScanEagle. Available online: http://www.boeing.com/boeing/defense-space/military/ scaneagle/ (accessed on 20 January 2015).

29. Textron Systems. Aerosonde. Available online: http://www.textronsystems.com/products/unmanned/ aerosonde (accessed on 20 January 2015). 
30. Northrop Grumman. Bat Unmanned Aircraft System (UAS). Available online: http://www. northropgrumman.com/Capabilities/BATUAS/Pages/default.aspx (accessed on 20 January 2015).

31. Shadow. Available online: http://www.textronsystems.com/products/unmanned/shadow (accessed on 20 January 2015).

32. Elbit Systems. Hermes 450 UAS. Available online: https://www.elbitsystems.com/elbitmain/areain 2 .asp? parent=3\&num=32\&num2=32 (accessed on 20 January 2015).

33. General Atomics Aeronautical. Predator B UAS. Available online: http://www.ga-asi.com/ products/aircraft/predator_b.php (accessed on 20 January 2015).

34. Global Hawk. Available online: http://www.northropgrumman.com/capabilities/globalhawk/Pages/ default.aspx (accessed on 20 January 2015).

35. Menthe, L.; Cordova, A.; Rhodes, C.; Costello, R.; Sullivan, J. The Future of Air Force Motion Imagery Exploitation. Technical Report 1133, RAND: Santa Monica, CA, USA, 2012.

36. Anderson, C. Fred Smith: FedEx wants UAVs. Available online: http://diydrones.com/profiles/ blogs/fred-smith-fedex-wants-uavs (accessed on 20 January 2015).

37. Google Buys Drone Company Titan Aerospace. Available online: http://www.businessinsider.com. au/google-buys-drone-company-titan-aerospace-2014-4 (accessed on 20 January 2015).

38. RQ-21A Blackjack. Available online: http://www.insitu.com/systems/integrator/rq-21a-blackjack (accessed on 20 January 2015).

39. STUAS/TIER II UAS. Available online: https://www.fbo.gov/index?s=opportunity\&mode=form\& id=e609fa4f0b50459089e3d4190adcd822\&tab=core\&_cview=1 (accessed on 20 January 2015).

40. FQM-151A. Available online: http://www.avinc.com/uas/adc/pointer/ (accessed on 20 January 2015).

41. Contract CR-003-15. Available online: http://www.defense.gov/Contracts/Contract.aspx? ContractID=5452 (accessed on 20 January 2015).

42. Grasmeyer, J.; Keennon, M. Development of the black widow micro air vehicle. Prog. Astronaut. Aeronaut. 2001, 195, 519-535.

43. Pathfinder Plus. Available online: http://www.dfrc.nasa.gov/Research/Erast/pathfinder.html (accessed on 20 January 2015).

44. Keennon, M.; Klingebiel, K.; Won, H.; Andriukov, A. Development of the nano hummingbird: A tailless flapping wing micro air vehicle. In Proceedings of the 50th AIAA Aerospace Science Meeting, Nashville, TN, USA, 9-12 January 2012.

45. Deng, S.; Percin, M.; van Oudheusden, B.; Remes, B.; Bijl, H. Experimental investigation on the aerodynamics of a bio-inspired flexible flapping wing micro Air vehicle. Int. J. Micro Air Veh. 2014, 6, 105-116.

46. Baek, S.; Ma, K.; Fearing, R. Efficient resonant drive of flapping-wing robots. In Proceedings of the IEEE/RSJ International Conference on Intelligent Robots and Systems (IROS 2009), St. Louis, MO, USA, 10-15 October 2009; pp. 2854-2860.

47. ROS. Available online: http://www.ros.org/ (accessed on 20 January 2015).

48. Carrigan, G.; Long, D.; Cummings, M.L.; Duffner, J. Human factors analysis of predator B crash. Available online: http://hdl.handle.net/1721.1/84129 (accessed on 22 April 2015). 
49. Revisiting the Reaper Revolution. Available online: http://nation.time.com/2012/02/27/1-thereaper-revolution-revisited/ (accessed on 20 January 2015).

50. NMSU's Flight Test Center Stages Successful UAV Test over Hatch, N.M. Available online: http://newscenter.nmsu.edu/Articles/view/7784 (accessed on 20 January 2015).

51. Unmanned Aerial Vehicles Group. Available online: http://www.uav.ethz.ch/ (accessed on 20 January 2015).

(C) 2015 by the author; licensee MDPI, Basel, Switzerland. This article is an open access article distributed under the terms and conditions of the Creative Commons Attribution license (http://creativecommons.org/licenses/by/4.0/). 